\title{
El uso de la tecnología de la información entre investigadores mexicanos: un estudio preliminar
}

\author{
CHARLOTTE Ford \\ School of Library and Information Science, Indiana \\ University Bloomington, IN 47405 \\ E-mail: ceford@indiana.edu \\ ANGÉliCa María Rosas GutiérReZ \\ Centro Universitario de Investigaciones Bibliotecológicas \\ Universidad Nacional Autónoma de México, 04510, México D.F., \\ Tel: (525)623-03-68 FAX: (525)550-74-61 \\ E-mail: rosas@ cuib.unam.mx
}

\begin{abstract}
RESUMEN
Este artículo presenta los resultados de una encuesta de científicos mexicanos (biólogos y físicos) realizada en 1998. Se envío a 400 investigadores un cuestionario con preguntas sobre el uso yla producción de fuentes deinformación impresas y electrónicas; sobre la disponibilidad y el uso de la tecnología de cómputo, y sobre los obstáculos percibidos en el uso de los recursos de cómputo. El 26.5\% contestó la encuesta. Se encontró que generalmente es bueno el acceso y el uso de las computadoras entre los investigadores mexicanos. Se notaron algunas diferencias individuales en cuanto al acceso y el uso de la tecnología de la información: los físicos, aquellos que tienen doctorados, y quienes trabajan en institutos de investigación tienen el mejor acceso a Internet. Se discuten las implicaciones de esto y se identifican áreas para futuros estudios.

Palabras clave: Tecnología de la información, Comportamiento informativo, Usuarios de computadoras

\section{USE OF INFORMATION TECHNOLOGY AMONG ACADEMC SCIEN- TISTSIN MEXICO: PRELIMINARY RESULTSOF A SURVEY Charlotte Ford Angélica Mańa Rosas-Gutiérmez}

Trabajo recibido el

28 de noviembre de 1999 *

Trabajo aceptado el 6 de enero de 2000

\section{ABST RACT}

This article reports the findings of a survey carried out in 1998 among Mexican biologists and physicists. The questionnaire, focusing on their use and production of print and electronic information sources; the availability and use of computer technology; and the obstacles they perceived in the use of these resources, was sent to 400 researchers (of which $26.5 \%$ responded). It was found that Mexican researchers generally have unencumbered and 
ample access to computer technology. Some differences in this access and use were identified from individual to individual and institution to institution. Physicists, researchers holding doctorate degrees, and those working in research institutes were found to enjoy optimal levels of access to and use of networked computer resources. The implications of these findings are discussed and areas for further study are identified. Key Words: Information Technology, Information Behaviord, Computers Users.

\section{INTRODUCCIÓN}

T a tecnología de la información y particularmente Internet, tienen cada vez mayor —trascendencia en la conducción y diseminación de la investigación científica. Algunas investigaciones han estudiado el uso que hacen los científicos de la tecnología dela información (e. g. Royal Society 1993; Bishop 1994; Philip 1995; Rolinson, Al-Shanbari y Meadows 1995; Cole y Bawden 1996; Abels, Liebscher y D enman 1996; Liebscher, Abels y D enman 1997; Hurd y Weller 1997). Sin embargo, poca atención se ha puesto al uso de la tecnología de la información por parte de los investigadores de países en desarrollo. Lo que sugieren los estudios realizados por Ehikhamenor (1990a, 1990b) y Nkewe (1995) en Nigeria; Cabrajecy D ukic (1991) en Croacia; Al-Shanbariy Meadows (1995) en Saudi Arabia, es poco uso de los recursos electrónicos tales como las bases de datos en línea; un gran uso del préstamo interbibliotecario, yun gran entusiasmo sobre el uso del correo electrónico para comunicarse internacionalmente.

El rápido crecimiento de la World Wide Web ha generado gran especulación en cuanto a las posibilidades de comunicación existentes para los investigadores que trabajan fuera de los E stados Unidos y Europa. Hay quienes afirman que Internet les ofrece a los científicos la posibilidad de compensar la escasez de recursos institucionales y les da la oportunidad de participar plenamente en la investigación internacional (Ginsparg 1997; Cronin y McKim 1996). Por el contrario, otros autores se preocupan y subrayan los costos de la infraestructura tecnológica y de la provisión de información, que pueden ser pro hibitivos para muchas instituciones en los países en vías de desarrollo, y sostienen que estos costos pueden impedir que la gente que trabaja en estas instituciones participe en la red académica y en la publicación (Elliott 1997; V outssas y Cetto 1996). El crecimiento de Internet en años recientes, en los países en vías de desarrollo ha sido impresionante,un ejemplo de esto es que para 1998 el número de sitios en Internet pertenecientes a América Latina, aumentó más rápido que el de cualquier otra región (Nua Internet Surveys 1998). En México, durante el periodo que va de 1995 a 1998, el número de servidores pasó de 7,000 a 80,000 (Network Wizards Survey 1998). Pero realmente es poco lo que se conoce acerca del uso que actualmente hacen de estas conexiones los investigadores que trabajan en los países de la región latinoamericana.

El presente artículo ofrece los resultados de un estudio realizado en 1998 en esta dirección. El propósito de este estudio fue determinar el acceso y uso de la tecnología delainformación entre biólogos y físicos que trabajan en universidades einstitutos de 
investigación en México. México es considerado un país en vías de desarrollo (Banco Mundial 1997) y, como tal, pertenece a la periferia científica. Sin embargo, dentro de esta "periferia" México es un país que ha desarrollado una importante infraestructura académica que lleva a cabo una considerable e importante producción científica (Statistical Y earbook for Latin America and the Caribbean 1997; UNE SCO Statistical Yearbook 1997; Krauskopf, Vera y Welljams-D orof 1995). Además, es un país que cuenta con un buen desarrollo bibliotecario y de servicios de información, una infraestructura fuerte de telecomunicaciones (Fernández de Zamora 1991; Lau 1993), y una visible presencia de Internet entre las universidades (lo cual sugiere un cierto nivel básico de conexiones entre las computadoras de los académicos) (ANUIES 1998). Además, el uso de las computadoras y las redes electrónicas parala producción, distribución y consumo de conocimiento científico parece ser generalizado. Así que hay mucho que aprender de la experiencia mexicana.

\section{ANTECEDENTES: LA IN VESTIGACIÓN CIENTÍFICA Y LA INFRAESTRUCTU- RA TECNOLÓGICA EN MÉXICO}

\section{El estado de la investigación científica en México}

La investigación en las universidades de América Latina es un fenómeno reciente, pues lo que tradicionalmente ha predominado en éstas ha sido la enseñanza. Aunque algunos centros de investigación científica fueron establecidos a principios del siglo X IX, el progreso real en la institucionalización de la investigación en las universidades sólo surgió en la primera mitad del siglo XX (Fortes y Lomnitz 1994). Sin embargo, el prestigio de la ciencia es todavía menor que el delaformación de profesionales. La investigación en México (como en toda América Latina) se inició sistemáticamente y en forma firme hasta hace pocos años, y pese al ingenio indígena y al espíritu aventurero español, no se conformó una tradición científica propiamente nacional, como sucedió en el caso de varios países europeos. Súmese a ello laignorancia pública del papel que juega la ciencia en la sociedad, y en muchos casos, la falta de apoyo institucional (Fortes y Lomnitz 1994; Magaña, Almeida, Carrollo y Torres 1995).

En México como en muchos países latinoamericanos, los profesores universitarios han sido por tradición catedráticos, es decir profesionales que enseñan pocas horas a la semana para lograr un prestigio social o para ofrecer un servicio público. Aunque la transformación de la educación superior en México ha permitido asalariar a profesores de tiempo completo, la mayoría de éstos son todavía de tiempo parcial y raramente realizan investigaciónes (O rnelas y Levy 1991). Mientras la investigación es vista como parte de la educación superior (particularmente de la educación superior pública), ésta no cuenta con un fuerte apoyo económico ni está efectivamente ligadaal desarrollo económico del país. Además una buena parte dela 
investigación que se realiza en México emana de institutos de investigación afiliados a universidades y de algunos institutos que están ubicados fuera de éstas (D e Ibarrola 1992; Ornelas y Levy 1991). La formación de profesionales es aún la función más importante de la educación superior; sin embargo, desde los años 70, se tomaron medidas para fortalecer la investigación, para lo cual fue notable la fundación del Consejo Nacional para la Ciencia y la Tecnología (CO NACYT) en 19701 y el establecimiento del Sistema Nacional de Investigadores (SNI) en 1984, gracias a los cuales los investigadores pueden duplicar sus ingresos de acuerdo con sus méritos (de Ibarrola 1992; Lau 1993).

El soporte para lainvestigación, desde el punto de vista de una infraestructura bibliotecaria, opera bajo un número de obstáculos en México, como sucede también en el resto de América Latina. Fernández de Zamora (1991), al estudiar datos de la IFLA y estadísticas nacionales y de la UNESCO, concluye que "América Latina aún no ha entrado a la sociedad de la información...Lainformación todavía no se ha convertido en un recurso estratégico parael desarrollo." Esta investigadora notó que las bibliotecas automatizadas y conectadas a bancos de datos internacionales coexisten al lado de bibliotecas que carecen de los recursos técnicos más básicos.

México es uno de los países más avanzados en América Latina por lo que toca a recursos bibliotecarios (Fernández de Zamora 1991) y a la producción de bases de datos bibliográficas (Rodríguez 1993, 1994). Sin embargo, la infraestructura bibliotecaria continúa plagada de problemas. Almada de Ascencio y Pérez de Almada (1991), especialistas en estudios dela información de la Universidad Nacional Autónoma de México (UNAM), describen las dificultades que existen para administrar un departamento de publicaciones seriadas en la UNAM, donde el 85\% de las revistas adquiridas son importadas. Las complicaciones incluyen regulaciones para los clientes, permisos de importación, carencia de moneda extranjera, inflación, servicio postal lento o ineficaz, costos de transporte, y esquemas problemáticos de precios. Las bibliotecas académicas fueron severamente afectadas por la recesión en los años 80, lo que provocó una disminución en los salarios y presupuestos reducidos para las bibliotecas así como para otros sectores de la educación superior en México (Fernández de Zamora 1990; O melas y Levy 1991).

México es un país importante en la producción de conocimiento científico en A mérica Latina. Tiene un gran número de investigadores e inversiones importantes que se suman a la investigación y el desarrollo (UNESCO 1997). Entre los países de A mérica Latina, Brasil, Argentina, México, Chile y Venezuela cuentan con más del 80\% de las publicaciónes regionales denominadas como mainstream(de reconocimiento internacional) (Krauskopf, Vera, Krauskopf y Welljams-D orof

1 En diciembre de 1970, en uno de los primeros actos de su gobierno, el presidente Luis Echeverría creó este Consejo, y desde entonces, dadas sus características y funciones, éste se ha constituido en el órgano guía principal de carácter nacional en materia de ciencia y tecnología.

http:/ / www.conacyt.mx 
1995). Las publicaciones científicas mexicanas están entre las publicaciones más altamente citadas del tercer mundo (O sareh y Wilson 1997).

Sin embargo México está todavía situado en la periferia de la ciencia global. Los investigadores mexicanos sufren de una escasa actualización de sus equipos, de dificultades para acceder a los desarrollos científicos y para asistir a reuniones internacionales e incluso nacionales, y tienen barreras de lenguaje (Lau 1993). Un estudio publicado en los años 1980 sobre ciencias de la salud, reveló que pocos artículos mexicanos son publicados en revistas de prestigio internacional, y que aquellos que son publicados tienen sólo un impacto modesto (Licea de Arenas 1992). Delgado y Russell (1992) estudiaron de 1978 a 1987 el impacto de los artículos indizados por el ISI y publicados por investigadores adscritos a la Universidad Nacional Autónoma de México (UNAM), y encontraron un incremento general en el nivel de producción de artículos, pero un bajo nivel de producción individual, así como también un bajo nivel de citación (en comparación con artículos de otros países publicados en las mismas revistas).

En años recientes, sin embargo, las políticas gubernamentales han impulsado la ciencia mexicana hacia la internacionalización. Los científicos mexicanos han sido estimulados, a través de los programas de subsidio, para publicar en revistas internacionales (Sistema Nacional de Investigadores 1998, Schoijet y Worthington 1993),y el número de artículos con al menos un autor mexicano indizado por el internacional SaieneCitationIndxx ha mostrado un aumento estable durante los últimos nueve años. Existen también evidencias de un incremento en la coautoría internacional entre científicos mexicanos de 1980 a 1990 (Russell 1995), y de que, hoy por hoy, prominentes científicos publican un porcentaje más alto de su trabajo en revistas internacionales (Russell 1998).

En conclusión, la ciencia mexicana está en la periferia de la ciencia mundial, pero México es un país sobresaliente de esta periferia y se están llevando a cabo serios esfuerzos para impulsar la participación de los científicos mexicanos en la ciencia internacional. Una pregunta pertinente es: ¿qué papel juega la tecnología de la información en este esfuerzo?

\section{La "conectividad" de los investigadores mexicanos y el uso de la tecnolo- gía de la información}

En las dos últimas décadas las redes de información se han establecido en América Latina con mucho éxito gracias al apoyo de varias organizaciones internacionales (Lau 1994) RED ALC (Red deAmérica Latina y el Caribe), patrocinado por la O EA y otras organizaciones, ha sido una red importante. CEPAL (la Comisión Económica para A mérica Latina) ha concebido y apoyado INFO PLAN, y la UNESCO ha apoyado la creación de varias redes y sistemas de información y del software MINISIS, el cual es ampliamente usado para el procesamiento estandarizado de intercambio de información en América Latina. Asimismo la IFLA, la FAO, y la OMS han también 
patrocinado importantes proyectos regionales (tales como la base de datos latinoamericana en bibliotecología y ciencias de la información INFO BILA; ${ }^{2}$ el sistema de información agrícola AG RINTER; ${ }^{3}$ y el Centro Latinoamericano y del Caribe de Información en Ciencias de la Salud BIREME). 4 Un programa regional para la cooperación entre redes de información y sistemas de información (INFO LAC) fuefundado en 1987 para coordinar proyectos de redes (Lau 1994).

La conexión de los países de América Latina a Internet se inició en el seno de las comunidades académicas y científicas en la década de 1980 (Cabezas 1995), y fue fomentada enormemente porlainiciativa de la Red Hemisférica Inter-Universitaria de Información Científica y Tecnológica ${ }^{5}$ (RedHUCyT) patrocinada por la OEA. Actualmente todos los países latinoamericanos están conectados (The Internet Society, 1996). A pesar de estos esfuerzos todavía existen impedimentos que dificultan la utilización de las redes. Los problemas comunes incluyen un limitado conocimiento tecnológico, recursos económicos escasos y, en algunos países, baja calidad de la infraestructura de telecomunicaciones y falta de capacitación (Lau 1994; Borman 1996).

Rodríguez $(1993,1994)$ se lamenta de las pocas bases de datos producidas en América Latina. Sin embargo, es importante notar, como ellamenciona, que México se encuentra a la vanguardia en materia de producción de bases de datos, así como de redes. La industria y el mercado del disco compacto en México es muy fuerte eincluye un gran número de productos académicos nacionales y gubernamentales (tales como las bases de datos producidas en la Universidad Nacional Autónoma de México y en la Universidad de Colima) (Levison e Iglesias 1993; Ainsworth 1994). Muchas de estas bases de datos están ahora disponibles a través de Internet ( $\mathrm{G}$ amboa 1998; Campos 1997).

Laautomatización debibliotecas en México se ha dado en gran medida en las universidades y bibliotecas especializadas, lo que no ha pasado ostensiblemente en otras bibliotecas debido a problemas presupuestarios (los cuales se dieron a raíz de las recesiones económicas que México padeció en los años 80, justamente cuando las aplicaciones de las microcomputadoras se estaban difundiendo ampliamente). D e acuerdo con Lau y Castro (1994), aproximadamente 20 sistemas de bibliotecas fueron totalmente automatizados en 1994; la mayoría de las universidades y bibliotecas especializadas habían automatizado sus servicios técnicos para esta época; cerca de 250 instituciones habían solicitado a la UNICEF copias del software de MicroIsis, y existían 10 nodos de Internet y Bitnet en el país.

2 La base de datos puede consultarse en el sitio http:/ / cuib.unam.mx

3 Para tener acceso a la base de datos en la web: http:/ / www.iica.cr/ agri200

4 Su página en la Web es http:/ / www.bireme.br

5 Su principal objetivo es conectar instituciones de los estados miembros a la Internet, integrando una red interoperable para el intercambio de información científica y técnica.

http:/ / www.oas.org/ SP/ PROG / RED / covers.htm o http:/ / www.redhucyt.oas.org/ webing 
D esde 1994, las conexiones a Internet y los recursos de información han proliferado en México. México tenía para 1998 un total de 83,948 hosts, cifra superada solamente por Brasil entre las naciones de América Latina (Network Wizards Intemet Survey1998). En la página Web de la ANUIE S (la A sociación Nacional de Universidades e Instituciones de Educación Superior) hay conexiones a más de 100 sitios Web deuniversidades, universidades técnicas e institutos de investigación, varios de los cuales ofrecen acceso a los catálogos de sus bibliotecas a través de Internet. Y el sistema de telecomunicaciones en México, que antes era un obstáculo significativo para acceder a las redes, ha sido recientemente modernizado (Tangeman 1996).

Sin embargo, existe poca información sobre el uso que hacen los investigadores mexicanos de las bases de datos en línea y CD-ROM, de los recursos bibliotecarios en línea y de Internet. Este estudio intenta llenar este vacío formulando y tratando de contestar las siguientes preguntas:

1. ¿Cuál es el grado de acceso y uso de la tecnología de información y los recursos de cómputo que tienen los investigadores científicos mexicanos?

2. ¿Qué diferencias significativas existen en el acceso y uso de los recursos de información electrónicos de acuerdo con la edad, el sexo, la categoría laboral, el grado de estudios, la disciplina y el tipo de afiliación institucional?

Examinar estas preguntas en el contexto mexicano puede tener amplias repercusiones. Específicamente, los resultados de este estudio pueden brindar nuevas percepciones sobre la clase de acceso que tienen los investigadores que trabajan actualmente en la "periferia" de la ciencia global en relación con Internet y los recursos en línea, y con el uso que hacen de estos recursos. Esto puede también ofrecer indicios sobre el impacto diferencial producido por el uso de Internet y otros recursos electrónicos entre los investigadores que trabajan en diferentes disciplinas e instituciones.

\section{METOdOLOGÍA}

Se seleccionaron los nombres de 400 biólogos y físicos mexicanos del directorio de investigadores y proyectos de investigación denominado ARIES, una base de datos desarrollada por la UNAM (disponible en http:/ / serpiente.dgsca.unam.mx/ dgia/ aries o http:/ / 132.248.9.9:8060). Se eligió a biólogos y físicos porque ellos conforman el grupo más grande de investigadores en México de acuerdo con las estadísticas del Sistema Nacional de Investigadores (Estadísticas Básicas 1997-1998).

Se eligió el directorio de ARIES después de un intento fallido por obtener una lista actualizada de los investigadores que integran el Sistema Nacional de Investigadores. ARIES proporciona información sobre las personas dedicadas a proyectos de investigación en 57 universidades mexicanas, incluyendo sus áreas de investigación y sus direcciones. El sistema permite hacer búsquedas de registros por proyecto 0 bien por investigador, pero el directorio presenta algunos problemas: los informes sobre los investigadores y los proyectos de investigación son reportados por las diferentes universidades y en algunos casos aparecen indiscriminadamente listados todos los miembros de facultades de ciertos departamentos, pero se proporciona 
muy poca información adicional sobre ellos, como por ejemplo sobre sus áreas de investigación. En otros casos, las universidades reportaron muy pocos investigadoresy algunas de las áreas de investigación listadas en el directorio no corresponden a la lista oficial de áreas UNESCO-CONACYT usadas por ARIES.

La vigencia de los registros también varía y algunas universidades actualizan sus registros con mayor frecuencia que otras. En el caso de la UNAM, la mayor parte de la información fue reciente (1997), pero en otras universidades la información no estaba actualizada: tenía fecha de 1993 a 1994.

Además el directorio en línea únicamente despliega 400 registros a la vez. No se pudo encontrar una solución a esta limitación incluso después de haber hablado con los responsables de la base de datos. Así pues, en el caso de las universidades que listaron más de 400 investigadores, los nombres de los investigadores que pertenecían a las áreas de biología y física (de acuerdo con la clasificación de la UNESCO-CONACYT), fueron sistemáticamente recuperados y transferidos. Es muy probable que algunos investigadores hayan sido inadvertidamente omitidos y que otras áreas de investigación hayan sido inadvertidamente incluidas. Se eliminaron los duplicadosy los nombres de investigadores que obviamente no pertenecían al área de física o biología. El listado final incluye un total de 2175 investigadores, delos cuales 1538 , o el 70\%, son biólogos, y 637, o el 30\%, son físicos; el 53\% de los investigadores pertenece a la UNAM y el $47 \%$ a otras instituciones. De este listado se seleccionaron al azar los nombres de 140 biólogos y 60 físicos de la UNAM, y de 140 biólogos y 60 físicos de otras universidades. Luego se tomaron y depuraron las direcciones de estos 400 investigadores.

Se desarrolló un cuestionario en español para obtener información de los biólogos y físicos mexicanos (véase apéndice A). Este cuestionario se estructuró principalmente en cuatro secciones:

1. Infomacónpessonal Incluye preguntas sobre la disciplina, el grado de estudios, la categoría laboral, la edad, el sexo, el tipo de institución, el tiempo dedicado a la investigación, y el énfasis institucional para publicary, particularmente, para publicar en revistas de alcance internacional.

2. Usoyproduccióndelosreaursosdeinfomacón Incluye preguntas sobre la participación en sociedades de carácter profesional tanto nacionales como internacionales; actividades de comunicación informal (impresos y publicaciones electrónicas), y actividades de comunicación formal (consumo y producción de investigaciones en formatos impresos y electrónicos de alcance nacional e internacional).

3. Disponibilidadyusodela computadara. Incluye preguntas so bre el acceso a computadoras, el correo electrónico e Internet, y la frecuencia de uso delas computadoras para las diferentes actividades relacionadas con el trabajo de investigación (incluyendo la comunicación internacional y nacional).

4. Peceppión delos dbstáailos para d usodelosrearsos decómputa Incluye preguntas sobre la calidad del acceso a las computadoras, el mantenimiento, el hardware y el 
software, la capacitación, el tiempo que se tiene para aprender el uso de los recursos, la conexión a las redes, etcétera.

Al finalizar las secciones del cuestionario se invitó a los investigadores para que compartieran cualquier comentario adicional que pudieran tener con relación al impacto que produce la tecnología de la información en su labor científica.

El cuestionario fue piloteado con físicos y biólogos en dos universidades, una de Estados Unidos y otra de México, en julio y agosto de 1998. Se enviaron cuatrocientos cuestionarios por correo en la primera semana de septiembre (1998), junto con cartas de introducción y sobres de retorno que ya tenían estampillas. Se recibieron ciento seis respuestas antes del 1ํㅡㄹ de diciembre (que corresponden al $26.5 \%$ del total). Los datos de las respuestas fueron capturados en SPSS para su análisis.

\section{RESULTADOS Y DISCUSIÓN}

Se obtuvieron respuestas de 24 físicos, 71 biólogos y 8 personas de otras disciplinas, más 3 personas que no reportaron disciplina, lo que representa un total de 106 respuestas. D e éstas 62 (58.5\%) manifiestan adscripción a la UNAM, y 44 (41.5 \%) pertenecen a otras instituciones. La mayoría de las personas que respondieron a los cuestionarios eran investigadores consumados. El encuestado típico dedica el 69\% del tiempo de trabajo a la investigación, pertenece a 3 sociedades profesionales, y ha asistido a 6 eventos profesionales durante los últimos tres años, al igual que lee regularmente 6 revistas impresas (5 extranjeras y 1 una mexicana) y ha producido numerosas publicaciones recientemente (véase tabla 1 ).

Tabla 1.

Promediodetrabajos repartados par tipodepublicacón para 1993-1998 (n=106)

\begin{tabular}{|l|c|}
\hline \multicolumn{1}{|c|}{ Tipo de publicación } & $\begin{array}{c}\text { Promedio } \\
\text { para } \\
\text { 1993-1998 }\end{array}$ \\
\hline Artículos en revistas impresas extranjeras & 5.16 \\
\hline Artículos en revistas impresas mexicanas & 2.48 \\
\hline Artículos en cualquier memoria de conferencia & 4.76 \\
\hline Informe para una agencia patrocinadora & 1.35 \\
\hline Capítulo en un libro académico & 1.08 \\
\hline Libro académico (editor o autor) & 0.51 \\
\hline
\end{tabular}




\section{A cceso y uso de los recursos electrónicos de información}

Según las mismas respuestas de aquellos investigadores que contestaron el estudio, el acceso a las computadoras y a Internet es bueno. Más del $90 \%$ de los encuestados tiene acceso a una computadora desde su oficina o laboratorio. Cerca del $80 \%$ tiene acceso al correo electrónico desde su oficina o laboratorio, y alrededor de las tres cuartas partes (76.2\%) tienen un acceso completo a Internet desde sus oficinas 0 laboratorios. Además, el $84 \%$ de las respuestas muestran que los investigadores tienen una computadora en sus casas, que más del $40 \%$ tiene acceso al correo electrónico también desde sus hogares, y que un 36.8\% tiene acceso completo a Internet desde sus hogares. (Véase tablas 2 y 3 )

Tabla 2.

Disponibilidady yacceso a la computadbra ya Intemet en e trabajo $(n=106)$

\begin{tabular}{|l|c|c|c|c|}
\hline \multirow{2}{*}{ Nivel de acceso } & \multicolumn{4}{|c|}{$\begin{array}{c}\text { Ubicación más cercana de una } \\
\text { computadora en el trabajo }\end{array}$} \\
\cline { 2 - 5 } & $\begin{array}{c}\text { En la oficina } \\
\text { y laboratorio }\end{array}$ & $\begin{array}{c}\text { Mismo } \\
\text { edificio }\end{array}$ & $\begin{array}{c}\text { Otro edificio } \\
\text { en el } \\
\text { campus }\end{array}$ & No sabe \\
\hline Computadora & $98(92.5 \%)$ & $5(5.7 \%)$ & $2(1.9 \%)$ & $0(0 \%)$ \\
\hline Correo electrónico & $83(79.0 \%)$ & $10(9.5 \%)$ & $12(11.4 \%)$ & $1 *$ \\
\hline $\begin{array}{l}\text { Conexión completa a } \\
\text { Internet }\end{array}$ & $80(76.2 \%)$ & $12(11.4 \%)$ & $13(12.4 \%)$ & $1 *$ \\
\hline * No se incluye en el porcentaje calculado (se trata de un individuo que \\
realizaba investigaciones en una estación biológica ubicada en el campo). \\
\hline
\end{tabular}

Tabla 3

Disponibilidady accesoa la computadara ya Inteme en $\mathrm{d}$ hogar $(\mathrm{n}=106)$

\begin{tabular}{|l|c|}
\hline \multicolumn{1}{|c|}{ Nivel de acceso } & $\begin{array}{c}\text { Núm. y \% de } \\
\text { encuesta dos }\end{array}$ \\
\hline Computadora en el hogar & $89(84.0 \%)$ \\
\hline Correo electrónico en el hogar & $45(42.5 \%)$ \\
\hline $\begin{array}{l}\text { Conexión completa a Internet desde el } \\
\text { hogar }\end{array}$ & $39(36.8 \%)$ \\
\hline
\end{tabular}


El uso de los recursos computarizados es muy variado (véase tabla 4). Por un lado, es impresionante que sólo 2 de 106 encuestados (1.9\%) no usen el correo electrónico. La utilización de otras clases de recursos es menos generalizada. El 25\% nunca usa la computadora para transferir archivos y cerca de otro $25 \%$ nunca hace búsquedas en catálogos en línea, así como una tercera parte tampoco hace búsquedas en los índices electrónicos. Los patrones de uso mostrados en la tabla 4 son muy similares a los patrones descritos en estudios norteamericanos y europeos: el procesador de textos fue la primera aplicación que tuvo gran acogida inicialmente, mientras que el uso de programas para análisis estadístico y de modelado de datos está menos difundido, aspecto que varía de acuerdo con la disciplina y las necesidades del investigador. En estos estudios el uso del correo electrónico también se distingue como el más importante que se ha hecho de las redes. La transferencia de archivos es menos frecuente, y la búsqueda en índices electrónicos y bancos de datos es aún menos frecuente (Rolinson, Al-Shanbari y Meadows 1996; Royal Society 1993; Morton \& Price 1988). Las estadísticas sobre el uso del correo electrónico, búsquedas en bases de datos electrónicos y la transferencia de archivos, son comparables con aquellas de universidades pequeñas de Estados Unidos a mediados de los años 90 (Abels, Liebscher y D enman 1996).

Tabla 4

Freaunia de uso delos reaursos dela computadara pera diferentes actividades (par parcentajederespursta)

\begin{tabular}{|l|c|c|c|c|c|}
\hline \multicolumn{1}{|c|}{$\begin{array}{c}\text { Actividad } \\
\text { [frecuencia } \\
\text { promedio] }\end{array}$} & $\begin{array}{c}\mathbf{5} \\
\text { Diario o } \\
\text { casi a } \\
\text { diario }\end{array}$ & $\begin{array}{c}\mathbf{4} \\
\text { Varias } \\
\text { veces a } \\
\text { la se- } \\
\text { mana }\end{array}$ & $\begin{array}{c}\mathbf{3} \\
\text { Varias } \\
\text { veces al } \\
\text { mes }\end{array}$ & $\begin{array}{c}\mathbf{2} \\
\mathbf{1} \text { vez al } \\
\text { mes } \\
\text { o menos }\end{array}$ & $\begin{array}{c}\mathbf{1} \\
\text { Nunca }\end{array}$ \\
\hline $\begin{array}{l}\text { Uso del procesador de } \\
\text { textos [4.75] }\end{array}$ & $84.0 \%$ & $11.3 \%$ & $1.9 \%$ & $0.9 \%$ & $1.9 \%$ \\
\hline $\begin{array}{l}\text { Análisis estadístico de } \\
\text { datos [2.98] }\end{array}$ & $15.5 \%$ & $18.4 \%$ & $25.2 \%$ & $30.1 \%$ & $10.7 \%$ \\
\hline $\begin{array}{l}\text { Simular 0 modelar datos } \\
\text { [2.38] }\end{array}$ & $10.5 \%$ & $14.7 \%$ & $14.7 \%$ & $22.1 \%$ & $37.9 \%$ \\
\hline $\begin{array}{l}\text { Uso del correo electróni- } \\
\text { co para comunicarse con } \\
\text { colegas en México [4.13] }\end{array}$ & $53.3 \%$ & $21.0 \%$ & $13.3 \%$ & $10.5 \%$ & $1.9 \%$ \\
\hline
\end{tabular}


52 Investigación Biblicteedóġca v. 13 N o. 27 julio/ diciembre de 1999

\begin{tabular}{|l|c|c|c|c|c|}
\hline \multicolumn{1}{|c|}{$\begin{array}{c}\text { Actividad } \\
\text { [frecuencia } \\
\text { promedio] }\end{array}$} & $\begin{array}{c}\mathbf{5} \\
\text { Diario o } \\
\text { casi a } \\
\text { dia rio }\end{array}$ & $\begin{array}{c}\mathbf{4} \\
\text { Varias } \\
\text { veces a } \\
\text { la se- } \\
\text { mana }\end{array}$ & $\begin{array}{c}\mathbf{3} \\
\text { Varias } \\
\text { veces al } \\
\text { mes }\end{array}$ & $\begin{array}{c}\mathbf{2} \\
\mathbf{1} \text { vez al } \\
\text { mes } \\
\text { o menos }\end{array}$ & $\begin{array}{c}\mathbf{1} \\
\text { Nunca }\end{array}$ \\
\hline $\begin{array}{l}\text { Uso del correo electróni- } \\
\text { co para comunicarse con } \\
\text { colegas en otros países } \\
\text { latinoamericanos [2.91] }\end{array}$ & $25.7 \%$ & $12.4 \%$ & $14.3 \%$ & $22.9 \%$ & $24.8 \%$ \\
\hline $\begin{array}{l}\text { Uso del correo electróni- } \\
\text { co para comunicarse con } \\
\text { colegas fuera de América } \\
\text { Latina [3.20] }\end{array}$ & $28.2 \%$ & $17.5 \%$ & $15.5 \%$ & $24.3 \%$ & $14.6 \%$ \\
\hline $\begin{array}{l}\text { "D ownload" 0 transfe- } \\
\text { rencia de archivos elec- } \\
\text { trónicos[2.60] }\end{array}$ & $10.6 \%$ & $20.2 \%$ & $12.5 \%$ & $31.7 \%$ & $25.0 \%$ \\
\hline $\begin{array}{l}\text { Búsquedas en catálogos } \\
\text { de biblioteca [2.50] }\end{array}$ & $8.7 \%$ & $10.7 \%$ & $26.2 \%$ & $31.1 \%$ & $23.3 \%$ \\
\hline $\begin{array}{l}\text { Búsquedas en bases de } \\
\text { datos electrónicos (como } \\
\text { Medline)[2.47] }\end{array}$ & $11.1 \%$ & $14.1 \%$ & $18.2 \%$ & $24.2 \%$ & $32.3 \%$ \\
\hline $\begin{array}{l}\text { Búsquedas en bancos de } \\
\text { datos [2.06] }\end{array}$ & $5.0 \%$ & $8.9 \%$ & $14.9 \%$ & $27.7 \%$ & $43.6 \%$ \\
\hline
\end{tabular}

Aunque el correo electrónico es ampliamente usado, el empleo de las computadoras por científicos mexicanos para otras actividades en red es menos frecuente (véase tabla 5). Por ejemplo, sólo cerca de una cuarta parte de los encuestados están suscritos a listas de discusión o grupos de interés. Mientras que el 70\% han distribuido (pre-prints) ${ }^{6}$ en los últimos 6 meses, sólo el 17\% lo ha hecho electrónicamente. El $40 \%$ de ellos había solicitado un pre-print a través del correo electrónico, y exactamente una tercera parte había transferido un pre-print o trabajo del Web en los últimos 6 meses. Estas cifras pueden parecer más bajas queaquellas queaparecen en estudios realizados en Estados Unidos e Israel paramediados de los años 90 . Una encuesta de 1994 hechaen Estados Unidos encontró queel 38\% de los biólogos yel 41\% delos físicos de universidades pequeñas estaban suscritos a grupos de discusión, y que el $46 \%$ de los biólogos y el 70\% de los físicos usaban la red para transferir archivos

6 El término pre-print indica la tirada preliminar de una parte o sección de un impreso, que generalmente se distribuye entre una comunidad académica o colegios invisibles para compartir, discutir o intercambiar ideas, metodologías o avances en las investigaciones. 
(Abels, Liebscher y D enman 1996). Un estudio efectuado en 1995 en Israel mostró que el $45 \%$ de los profesores de la Hebrew University estaba suscrito a grupos de discusión, y el 48\% usaba Internet parala transferencia de archivos (Lazinger, Bar-Ilan y Peritz 1997). Sin embargo, comparar esta clase de estadísticas presenta problemas de validez, pues la manera en que se formularon las preguntas puede alterar las respuestas (por ejemplo a los encuestados en México se les preguntó si en los últimos seis meses habían distribuido y transferido archivos)

Tabla 5.

Usoy distribuiaón delos rearsos deetrónicos deinfomaión enlos últimos 6 meses

\begin{tabular}{|l|c|}
\hline \multicolumn{1}{|c|}{ Actividad } & $\begin{array}{c}\text { Núm. y \% de encuesta dos } \\
\text { con respuestas a firmativas }\end{array}$ \\
\hline $\begin{array}{l}\text { Colaboración con colegas mediante el correo } \\
\text { electrónico }\end{array}$ & $63(61.2 \%)$ \\
\hline $\begin{array}{l}\text { Solicitud de pre-prints a través del correo elec- } \\
\text { trónico }\end{array}$ & $40(38.5 \%)$ \\
\hline Transferencia de pre-prints por Internet & $34(33.0 \%)$ \\
\hline $\begin{array}{l}\text { Lectura habitual de alguna lista de discusión } \\
\text { electrónica* }\end{array}$ & $23(22.5 \%)$ \\
\hline $\begin{array}{l}\text { Distribución de pre-prints o copias de su traba- } \\
\text { jo en forma electrónica }\end{array}$ & $19(18.3 \%)$ \\
\hline * Esta pregunta fue planteada en general, y no para un lapso de seis meses \\
\hline
\end{tabular}

El uso de Web para diseminar los resultados de la investigación es sumamente bajo entre los científicos: menos del 20\% de los encuestados tiene una página en la Web, y sólo 5 personas mencionaron que tienen copias de sus trabajos en la Web. Pocos leen revistas electrónicas (sólo una cuarta parte) y algunos comentaron que no hay revistas electrónicas de interés en su área. Por otro lado, la utilización de revistas impresas especialmente extranjeras es señalado como extremadamente importante. El 98\% de los encuestados calificó a las revistas impresas extranjeras como "muy importantes" o "importantes", fueron éstas las fuentes más favorecidas, seguidas por los libros, las publicaciones de crítica y revisión (review), y las memorias de conferencia impresas. Las revistas y conferencias electrónicas fueron calificadas como las fuentes menos valiosas (véase la tabla 6). 
Tabla 6.

Impatancia delos reaursos deinformación impresosy deetrónicos para la investigacoón (por parcentajederespursta)

\begin{tabular}{|l|c|c|c|c|c|}
\hline \multicolumn{1}{|c|}{$\begin{array}{c}\text { Recurso } \\
\text { [importancia prome- } \\
\text { dio] }\end{array}$} & $\begin{array}{c}\mathbf{5} \\
\text { Muy impor- } \\
\text { ta nte }\end{array}$ & $\begin{array}{c}\mathbf{4} \\
\text { Impor- } \\
\text { tante }\end{array}$ & $\begin{array}{c}\mathbf{3} \\
\text { Modera- } \\
\text { damente } \\
\text { impor- } \\
\text { tante }\end{array}$ & $\begin{array}{c}\mathbf{2} \\
\text { No muy } \\
\text { impor- } \\
\text { tante }\end{array}$ & $\begin{array}{c}\mathbf{1} \\
\text { No } \\
\text { uso }\end{array}$ \\
\hline $\begin{array}{l}\text { Revistas impresas extran- } \\
\text { jeras [4.84] }\end{array}$ & $85.7 \%$ & $12.4 \%$ & $1.9 \%$ & $0 \%$ & $0 \%$ \\
\hline $\begin{array}{l}\text { Revistas impresas mexi- } \\
\text { canas [3.38] }\end{array}$ & $23.1 \%$ & $27.9 \%$ & $22.1 \%$ & $17.3 \%$ & $9.6 \%$ \\
\hline $\begin{array}{l}\text { Revistas electrónicas } \\
\text { [2.75] }\end{array}$ & $19.2 \%$ & $17.2 \%$ & $15.2 \%$ & $16.2 \%$ & $32.3 \%$ \\
\hline $\begin{array}{l}\text { Preprints electrónicos } \\
\text { [2.91] }\end{array}$ & $21.1 \%$ & $23.2 \%$ & $12.6 \%$ & $11.6 \%$ & $31.6 \%$ \\
\hline Libros [4.17] & $39.2 \%$ & $44.1 \%$ & $12.7 \%$ & $2.0 \%$ & $2.0 \%$ \\
\hline $\begin{array}{l}\text { Publicaciones de crítica y } \\
\text { revisión ("review") [3.89] }\end{array}$ & $37.0 \%$ & $37.0 \%$ & $13.0 \%$ & $4.0 \%$ & $9.0 \%$ \\
\hline $\begin{array}{l}\text { Memorias de conferencia } \\
\text { impresas [3.61] }\end{array}$ & $19.4 \%$ & $39.8 \%$ & $26.2 \%$ & $11.7 \%$ & $2.9 \%$ \\
\hline $\begin{array}{l}\text { Memorias de conferencia } \\
\text { electrónicas [2.52] }\end{array}$ & $8.3 \%$ & $22.9 \%$ & $18.8 \%$ & $12.5 \%$ & $37.5 \%$ \\
\hline $\begin{array}{l}\text { Índices y resúmenes im- } \\
\text { presos [3.41] }\end{array}$ & $21.0 \%$ & $37.0 \%$ & $18.0 \%$ & $10.0 \%$ & $14.0 \%$ \\
\hline $\begin{array}{l}\text { Índices y resúmenes } \\
\text { electrónicos [3.26] }\end{array}$ & $32.7 \%$ & $22.4 \%$ & $10.2 \%$ & $7.1 \%$ & $27.6 \%$ \\
\hline $\begin{array}{l}\text { Ficheros de biblioteca } \\
\text { [3.13] }\end{array}$ & $13.7 \%$ & $28.4 \%$ & $31.4 \%$ & $9.8 \%$ & $16.7 \%$ \\
\hline $\begin{array}{l}\text { Catálogos electrónicos } \\
\text { de biblioteca [3.32] }\end{array}$ & $30.7 \%$ & $27.7 \%$ & $8.9 \%$ & $7.9 \%$ & $24.8 \%$ \\
\hline
\end{tabular}


Al parecer, las fuentes de información impresas continúan siendo una parte importante para la investigación de los científicos encuestados. Incluso entre los recursos secundarios de información, los índices impresos obtuvieron un voto más favorable que los índices electrónicos. Sólo los catálogos electrónicos de las bibliotecas superaron a los ficheros tradicionales.

El advenimiento de la Web no ha cambiado la primacía que las revistas impresas de alcance internacional tienen en relación al desarrollo de la ciencia, ni aparentemente sugiere un cambio en los patrones de colaboración internacional: los científicos mexicanos usan el correo electrónico con más frecuencia para comunicarse con sus colegas fuera de América Latina, que con sus colegas de otros países latinoamericanos (tabla 4). Un comentario hecho en las respuestas fue: "En particular con América Latina la comunicación es poca, por no decir nula. Para mí implica mucho tiempo buscar en Internet acerca de investigadores que trabajen en mi área."

Al final del cuestionario se preguntó sobre los obstáculos que presenta el uso de la computadora. Los encuestados en general se mostraron muy satisfechos con el acceso a las computadoras, un 85\% de ellos calificó el acceso como "excelente" o "bueno." La instrucción en el uso de la computadora y el apoyo técnico recibieron calificaciones bajas; el mayor obstáculo notado fue la disponibilidad del tiempo para aprender sobre el uso de los recursos de cómputo (el cual fue calificado como "malo" por el 30\% de los encuestados) (véase tabla 7).

Tabla 7.

\section{Calificacón delos factores ténicos}

\begin{tabular}{|l|c|c|c|c|c|}
\hline \multicolumn{1}{|c|}{ Factor técnico } & Excelente & Bueno & $\begin{array}{c}\text { Satis- } \\
\text { factorio }\end{array}$ & Malo & No sé \\
\hline Acceso a computadoras & $51.9 \%$ & $33.0 \%$ & $12.3 \%$ & $2.8 \%$ & $0 \%$ \\
\hline $\begin{array}{l}\text { Mantenimiento de computado- } \\
\text { ras }\end{array}$ & $24.5 \%$ & $32.1 \%$ & $22.6 \%$ & $20.8 \%$ & $0 \%$ \\
\hline Equipo (hardware) adecuado & $22.9 \%$ & $37.1 \%$ & $23.8 \%$ & $14.3 \%$ & $1.9 \%$ \\
\hline Programas (software) adecuados & $23.8 \%$ & $37.1 \%$ & $27.6 \%$ & $10.5 \%$ & $1.0 \%$ \\
\hline $\begin{array}{l}\text { Instrucción sobre el uso de la } \\
\text { computadora }\end{array}$ & $14.2 \%$ & $27.4 \%$ & $34.9 \%$ & $20.8 \%$ & $2.8 \%$ \\
\hline $\begin{array}{l}\text { Ayuda y apoyo sobre el manejo } \\
\text { de la computadora }\end{array}$ & $17.3 \%$ & $27.9 \%$ & $38.5 \%$ & $15.4 \%$ & $1.0 \%$ \\
\hline $\begin{array}{l}\text { Tiempo disponible para aprender } \\
\text { sobre los recursos de cómputo }\end{array}$ & $3.8 \%$ & $22.1 \%$ & $41.3 \%$ & $29.8 \%$ & $2.9 \%$ \\
\hline
\end{tabular}


Investigación Biblidtecolóġa V. 13 N o. 27 julio/ diciembre de 1999

\begin{tabular}{|l|c|c|c|c|c|}
\hline \multicolumn{1}{|c|}{ Factor técnico } & Excelente & Bueno & $\begin{array}{c}\text { Satis- } \\
\text { factorio }\end{array}$ & Malo & No sé \\
\hline Ubicación de las impresoras & $34.9 \%$ & $28.3 \%$ & $21.7 \%$ & $14.2 \%$ & $0.9 \%$ \\
\hline Calidad de las impresoras & $36.8 \%$ & $27.4 \%$ & $22.6 \%$ & $12.3 \%$ & $0.9 \%$ \\
\hline Acceso a las redes & $29.2 \%$ & $34.9 \%$ & $23.6 \%$ & $11.3 \%$ & $0.9 \%$ \\
\hline $\begin{array}{l}\text { Calidad de las conexiones a las } \\
\text { redes }\end{array}$ & $23.1 \%$ & $31.7 \%$ & $28.8 \%$ & $13.5 \%$ & $2.9 \%$ \\
\hline
\end{tabular}

La necesidad de contar con más tiempo y capacitación se reflejó en algunos comentarios. Por ejemplo, un investigador enfatizó que: "En México es necesario mayor información sobre el potencial y uso de la tecnología de la informática. Considero que ésta ha sido subutilizada". O tro escribió: "No es fácil que alguien tenga la paciencia de enseñarle a uno a usar las cosas." Comentarios so bre la falta de tiempo incluyen el siguiente: "Considero que esta tecnología es excelente, lo que nos falta es tiempo para estar al día, ya que la investigación con apoyo de microscopios ópticos y/ o electrónicos requiere todo mi tiempo, así como la redacción de artículos." O tro investigador señaló: "Me gustaría poder utilizar más ampliamente toda la tecnología de información por computadora ya que el tiempo es nuestro peor enemigo."

Sin embargo, los obstáculos más frecuentemente mencionados estuvieron relacionados con el costo de la tecnología de la información. A continuación se citan algunos de estos comentarios:

* La principal limitación en el acceso a bases de datos o revistas vía Internet es el costo.

* Los investigadores [de las instituciones estatales] cada día contamos con menos recursos como para contratar los servicios electrónicos adecuados.

* Es difícil para las instituciones de gobierno apoyar la compra del software y hardware para actualizarse en las nuevas versiones.

* El software avanza más rápido que los recursos.

* Larazón de no usar bancos de datos, e.g., para buscarcitas, artículos... es que es demasiado caro y las bibliotecas aquí tienen el uso muy restringido. Esa es una desventaja muy grande en México y supongo que en Latinoamérica en general.

Estos comentarios, y los asuntos relativos a la pobre calidad de las conexiones a las redes en algunas áreas, sugieren que los investigadores en México están todavía sujetos a limitaciones como resultado de su estatus laboral, como sucede en cualquier otro país en vías de desarrollo, a pesar de que tienen un amplio acceso a muchos recursos de información electrónicos. Sin embargo, el acceso y uso de la tecnología de la información parece ser bueno, y hubo también muchos comentarios favorables en el cuestionario que relacionaba el impacto de la tecnología con el trabajo científico, como por ejemplo: "D efinitivamente el impacto de la tecnología de 
lainformación en nuestro trabajo deinvestigación es una herramienta que ha contribuido a potenciar la capacidad, alcance, difusión e intercambio de nuestra información de una forma sustancial y determinante," y "Ésta ha hecho más rápido y fácil el intercambio de información, para el aprendizaje de temas de interés, y para mantenerse actualizado." Adjetivos tales como "excelente", "maravilloso" y, sobre todo, "indispensable" se usaron una y otra vez para describir la tecnología de la información.

\section{D iferencias en el acceso y uso de los recursos de información electrónicos de acuerdo con la edad, el sexo, el grado, la categoría laboral y el nivel educativo}

Estudios previos estudios sobre el uso de la tecnología de la información han encontrado diferencias sistemáticas con base en factores como edad, nivel laboral, y nivel educativo (Royal So ciety 1993, Rolinson, Al-Shanbari y Meadows 1995; Lazinger, Bar-llan y Peritz 1997). Se realizaron tablas cruzadas y pruebas chi-cuadrada, así como pruebas T de muestras independientes y ANOVA (análisis de varianza) según la conveniencia, para determinar cualquier diferencia significativa con respecto a las variables mencionadas.

Los investigadores contestaron de acuerdo alos rangos de edad (21-30, 31-40, etcétera.) Sólo 2 de ellos tenían 30 años o menos, así que éstos no fueron incluidos en el análisis.

Tabla 8.

Edad delosinvestigadares $(n=104)$

\begin{tabular}{|c|c|}
\hline Rango de edad & Núm. y $\%$ de encuesta dos \\
\hline $21-30$ años & $2(1.9 \%)$ \\
\hline $31-40$ años & $29(27.9 \%)$ \\
\hline $41-50$ años & $48(46.2 \%)$ \\
\hline $51-60$ años & $19(18.3 \%)$ \\
\hline 60 años & $6(5.8 \%)$ \\
\hline
\end{tabular}

La prueba de ANOVA se aplicó para mensurar el posible efecto de la edad (agrupadas por décadas) en las mediciones sobre el uso de los recursos de información electrónicos, pero no se encontraron diferencias significativas, excepto que los investigadores de mayor edad contestaron que usan muy poco el procesador detextos 
( $\mathrm{df}=3.98 ; \mathrm{F}=5.427 ; \mathrm{p} 01)$. En relación con la edad se encontró una diferencia significativa en los patrones de publicación en los últimos 5 años: la publicación de artículos en revistas científicas y libros se incrementa con la edad. Los investigadores entre el rango de los 50 años eran más prolíficos para escribir artículos, y aquellos entre el rango de los 60 años para escribir o editar libros (tabla 9).

Tabla 9.

Promedios detipos dedbumentopublicados en los últimos 5 años, respuesta par rango deeelad

\begin{tabular}{|l|c|c|c|c|}
\hline \multicolumn{1}{|c|}{ Rango de edad } & $\begin{array}{c}\text { Artículos en } \\
\text { revistas } \\
\text { mexicanas }\end{array}$ & $\begin{array}{c}\text { Artículos en } \\
\text { revistas } \\
\text { extranjeras }\end{array}$ & $\begin{array}{c}\text { Total de } \\
\text { artículos } \\
\text { en revistas }\end{array}$ & Libros \\
\hline $31-40$ años & 2.3 & 3.6 & 5.9 & 0.35 \\
\hline $41-50$ años & 2.0 & 4.1 & 6.1 & 0.44 \\
\hline $51-60$ años & 4.3 & 10.1 & 14.4 & 0.68 \\
\hline 60 años & 1.2 & 4.2 & 5.4 & 1.6 \\
\hline
\end{tabular}

El sexo ha sido considerado también como un posible factor de influencia en el uso delos recursos de información. Setenta y cinco (72.8\%) de las respuestas fueron de hombresy 28 (27.2\%) demujeres. En el presente estudio se encontraron muy pocas diferencias significativas. En promedio, las mujeres usan más frecuentemente las computadoras para comunicarse por correo electrónico (aunque no es significativo) y para hacer búsquedas en bases de datos (significativo en un nivel de .05, $\mathrm{df}=94)$. Ellas las usan en menor grado para modelar o simular datos ( $\mathrm{df}=55, \mathrm{p} 01)$. Las mujeres también publican pocos artículos en revistas internacionales (un promedio de 2.8 artículos en los últimos 5 años, en contraposición con los hombres cuyo promedio es de 6.0). Sin embargo estas diferencias pueden tener más relación con las disciplinas en las cuales ellas trabajan, que con el sexo al que pertenecen: de todos los investigadores que contestaron, sólo tres mujeres fueron del área de física, y (como se discute más tarde), los biólogos producen un menor porcentaje de publicaciones internacionales y usan la computadora muy poco para el modelado de datos, a diferencia de lo que hacen los físicos.

El nivel educativo y la posición laboral también fueron considerados como posibles factores que influyen en los investigadores en cuanto al uso de los recursos electrónicos de información. La mayor parte de las respuestas correspondieron a investigadores que cuentan con grados de maestría o doctorado (véase tabla 10). La mayoría de los encuestados también eran o titulares o asociados, aunque unos pocos eran técnicos (véase tabla 11). 
Tabla 10.

Gradoeeducativo $(n=104)$

\begin{tabular}{|l|c|}
\hline \multicolumn{1}{|c|}{ Grado } & $\begin{array}{c}\text { Núm. y \% } \\
\text { de respuestas }\end{array}$ \\
\hline D octorado & $58(55.8 \%)$ \\
\hline Maestría & $37(35.6 \%)$ \\
\hline Licenciatura & $8(7.7 \%)$ \\
\hline Otros & $1(1.0 \%)$ \\
\hline
\end{tabular}

Tabla 11.

Cateegríalabaral

\begin{tabular}{|l|c|}
\hline \multicolumn{1}{|c|}{ Categoría } & $\begin{array}{c}\text { Núm. y \% } \\
\text { de respuestas }\end{array}$ \\
\hline Titular & $74(71.2 \%)$ \\
\hline Asociado & $21(20.2 \%)$ \\
\hline Técnico & $8(7.7 \%)$ \\
\hline O tros & $1(1.0 \%)$ \\
\hline
\end{tabular}

Los niveles laborales y educativos no estaban fuertemente correlacionados uno con el otro (correlación Pearson $=0.156$, no es significativo a un nivel .05). Esto sugiere que ambos factores deberían ser examinados.

Se encontraron pocas diferencias significativas en relación con el nivel laboral por lo que toca al uso de la tecnología de la información. Es interesante notar que era más probable que los titulares distribuyeran más pre-prints impresos que los asociados (cerca del 80\% lo hizo en los últimos 6 meses, en oposición al 50\% de los asociados y técnicos), pero no hubo una diferencia significativa en sus patrones de distribución electrónica (18\% de los titulares y $24 \%$ de los asociados distribuyeron pre-prints electrónicos). También resultó menos probable que los titulares leyeran listas de discusión: solamente el 17\% lo hizo, en contraste con el 38\% de los asociados. En general, las diferencias en cuanto a uso de la tecnología de la información fueron mínimas.

Sin embargo se encontraron algunas diferencias notables relacionadas con el nivel educativo. La prueba de ANO VA sugirió diferencias en los patrones de publicación, así como diferencias en muchos aspectos vinculados con del uso de la computadora (tales como el procesador de textos, el correo electrónico, la transferencia de archivos, y la búsqueda de información en catálogos electrónicos y bases de datos). Como el 90\% de quienes contestaron tienen el grado de maestro o doctor, se realizaron pruebas T de muestras independientes para comparar estos dos niveles educativos. Aquellos que tienen grado de doctor dedican más tiempo a la investigación, sienten más presión para publicar (y particularmente para publicar en revistas extranjeras), pertenecen a más asociaciones internacionales, y asisten a más reuniones internacionales, y también publican más artículos en revistas de carácter internacional (véase la tabla 12). 
Tabla 12.

Adividades deinvestigadón par nived educativo(n=95)

\begin{tabular}{|l|c|c|}
\hline \multicolumn{1}{|c|}{ Actividad } & \multicolumn{2}{|c|}{ Mayor nivel educa tivo } \\
\cline { 2 - 3 } & Maestría & Doctorado \\
\hline \% de tiempo dedicado a la investigación * & 64.3 & 74.2 \\
\hline Núm. de membresías a una asociación internacional** & 0.8 & 2.2 \\
\hline $\begin{array}{l}\text { Núm. de asistencias a reuniones internacionales } \\
\text { (últimos 3 años)** }\end{array}$ & 1.3 & 2.8 \\
\hline $\begin{array}{l}\text { Núm. de artículos publicados en revistas extranjeras } \\
\text { (últimos 5 años)** }\end{array}$ & 2.2 & 7.7 \\
\hline$* P<05$ & & \\
$* *$ p $>.01$ & & \\
\hline
\end{tabular}

También se encontraron diferencias significativas en cuanto al uso de la computadora entre estos dos grupos de investigadores: aquellos con grado de doctor tenían mayor acceso al correo electrónico y en general a Internet desde sus oficinas o laboratorios $\left(\mathrm{X}^{2}=5.65, \mathrm{p}<05 ; \mathrm{X}^{2}=5.76, \mathrm{p}<05\right)$, y también mayor acceso al correo electrónico desde sus hogares $\left(\mathrm{X}^{2}=4.36, \mathrm{p}<05\right)$. No es por tanto sorprendente que ellos usen el correo electrónico más frecuentemente para comunicarse con sus colegas dentro del país y fuera de América Latina. Ellos también transfieren archivos más frecuentemente ( $\mathbf{d} \mathbf{f}=91 \mathrm{p}<=001)$, y es más probable que tengan una página en la Web: cerca de una cuarta parte de quienes tienen doctorado tienen su página, mientras que menos del $6 \%$ de quienes tienen maestría tenían una $\left(\mathrm{X}^{2}=4.97, \mathrm{p}<05\right)$.

\section{D iferencias en cuanto al acceso y uso de los recursos de información elec- trónicos por disciplina y por tipo de institución}

Los datos fueron también examinados para encontrar diferencias relacionadas con su disciplina entre los físicos y biólogos (24 y 71 respuestas, respectivamente) usando una prueba $\mathrm{T}$ de muestras independientes. Como grupo, los físicos parecen mejor conectados con la comunidad científicainternacional y sienten más presión para publicar en revistas del extranjero $(d f=93, p<01)$ y por ende, publican más artículos en revistas extranjeras $(\mathbf{d f}=90, \mathrm{p}<01)$. D e hecho los físicos han publi- 
cado en promedio 9 artículos en revistas extranjeras en los últimos 5 años, en comparación con un promedio de 3.8 por parte de los biólogos. Los biólogos publican más artículos en revistas mexicanas (un promedio de 2.8 artículos comparado con 1.7 por los físicos)y también leen más revistas mexicanas (un promedio de 1.4 revistas, mientras que los físicos leen 0.58 de revistas mexicanas), aunquelos primeros no leen menos revistas extranjeras (véase la tabla 13).

Tabla 13.

Patrones delectura ypublicacón: físios $(\mathrm{n}=24)$ ybidogos $(\mathrm{n}=71)$

\begin{tabular}{|l|c|c|}
\hline & Físicos & Biólogos \\
\hline Núm. de revistas extranjeras regularmente leídas & 4.6 & 5.1 \\
\hline Núm. de revistas mexicanas regularmente leídas* & 0.6 & 1.4 \\
\hline Artículos publicados en revistas extranjeras** & 9.0 & 3.8 \\
\hline Artículos publicados en revistas mexicanas & 1.7 & 2.8 \\
\hline $\begin{array}{l}* \text { p .05 } \\
\text { ** p .01 }\end{array}$ & \\
\hline
\end{tabular}

El uso de la tecnología electrónica por parte de físicos y biólogos presentó algunas diferencias. Los físicos son mucho más asiduos a distribuir pre-prints electrónicos: $41.2 \%$ de los físicos lo hacen, en comparación con el 11.3\% de los biólogos. La prueba T de muestras independientes, mostró que los físicos usan la computadora más frecuentemente que los biólogos para transferir archivos ( $d \mathbf{f}=91, \mathrm{p}<01)$ y para modelar o simular datos ( $\mathrm{d} f=82, \mathrm{p}<001)$. Mientras que ambos grupos tienen amplio acceso a las computadoras, los físicos tienen un mejor acceso al correo electrónico y en general a Internet en el trabajo: 92\% de ellos tienen acceso al correo electrónico y a Internet en sus oficinas o laboratorios. El acceso al correo electrónico desciende para los biólogos al 72.9\% mientras que el $70 \%$ tiene acceso completo a Internet en sus oficinas o laboratorios. Sin embargo, el acceso a las redes de computadoras fue igualmente positivo para ambos grupos.

Los investigadores mexicanos pueden estar afiliados a instituciones de investigación o departamentos de universidades. ${ }^{7} \mathrm{El}$ 62\% de las respuestas indicaba que los investigadores trabajaban en institutos de investigación, y el 38\% en departamentos

7 La clasificación de institutos de investigación o departamentos de universidades fue hecha por los mismos investigadores. 
de universidades. No es sorprendente que existan diferencias entre los científicos que trabajan en estos distintos ambientes. Aquellos que trabajan en institutos de investigación dedican, en promedio, alrededor de tres cuartas partes de su tiempo a la investigación, mientras que sus colegas de los departamentos universitarios invierten menos del $60 \%$ de este tiempo. Los científicos que trabajan en institutos sienten más presión para publicar, y particularmente para publicar en revistas extranjeras ( $d f=101, p<001)$. A unque ambos grupos mostraron grandes similitudes en sus hábitos de lectura y en la importancia que le dan a los diferentes recursos de información, los investigadores afiliados a institutos de investigación habían publicado más del doble en revistas extranjeras, y más del doble de capítulos de libros en losúltimos 5 años (tabla 14).

Tabla 14

Patrones depublicaión en los últimos 5 años deacuedb a institutosy departamentos deuniversidades $(\mathrm{n}=39)$

\begin{tabular}{|l|c|c|}
\hline & Institutos & Departamentos \\
\hline $\begin{array}{l}\text { Artículos publicados en revistas extranje- } \\
\text { ras* }\end{array}$ & 6.5 & 3.0 \\
\hline Artículos publicados en revistas mexicanas & 2.3 & 2.7 \\
\hline Capítulos de libros* & 1.4 & 0.6 \\
\hline Libros & 0.5 & 0.5 \\
\hline *significativo 0.5 nivel (df=98) & & \\
\hline
\end{tabular}

El acceso y uso de las computadoras varió entre institutos y departamentos universitarios. Alrededor del 85\% de quienes trabajan en departamentos tienen computadoras en sus laboratorios y oficinas, mientras que la cifra para quienes pertenecen a institutos es del 98.4\%. El acceso al correo electrónico y a Internet en general fue mejor para aquellos investigadores que trabajan en institutos de investigación: nueve décimas partes de éstos tienen acceso desde sus oficinas, mientras que sólo tres cuartas partes de quienes laboran en universidades tienen un acceso comparable $\left(\mathrm{X}^{2}=12.34\right.$ para el correo electrónico, $\mathrm{X}^{2}=10.74$ para el acceso a la Internet; $\mathrm{p}<.001$ en ambos casos). 
Tabla 15.

Nive deaccsoa las computadaras entrelosenuestadbs afiliados a instituriones deinvesticacióny departamentos universitarios

\begin{tabular}{|l|c|c|c|}
\hline \multirow{2}{*}{ Nivel de acceso } & \multicolumn{3}{|c|}{$\begin{array}{c}\text { Ubicación más cercana de una } \\
\text { computadora en los institutos }\end{array}$} \\
\cline { 2 - 4 } & $\begin{array}{c}\text { Oficina o la- } \\
\text { boratorio }\end{array}$ & $\begin{array}{c}\text { Mismo } \\
\text { edificio }\end{array}$ & $\begin{array}{c}\text { Otro edificio } \\
\text { en el campus }\end{array}$ \\
\hline Computadoras & $98.4 \%$ & $1.6 \%$ & $0 \%$ \\
\hline $\begin{array}{l}\text { Computadoras con conexión al co- } \\
\text { rreo electrónico }\end{array}$ & $90.5 \%$ & $7.9 \%$ & $1.6 \%$ \\
\hline \multirow{2}{*}{$\begin{array}{l}\text { Computadoras con conexión comple- } \\
\text { ta a Internet }\end{array}$} & $87.3 \%$ & $9.5 \%$ & $3.2 \%$ \\
\hline \multirow{2}{*}{ Nive acceso } & \multicolumn{2}{|c|}{$\begin{array}{c}\text { Ubicación más cercana de una } \\
\text { computadora en los deparatmentos }\end{array}$} \\
\cline { 2 - 4 } & $\begin{array}{c}\text { Oficina o la- } \\
\text { boratorio }\end{array}$ & $\begin{array}{c}\text { Mismo } \\
\text { edificio }\end{array}$ & $\begin{array}{c}\text { Otro edificio el campus } \\
\text { en el }\end{array}$ \\
\hline Computadoras & $84.6 \%$ & $12.8 \%$ & $0.3 \%$ \\
\hline $\begin{array}{l}\text { Computadoras con conexión al co- } \\
\text { rreo electrónico }\end{array}$ & $61.5 \%$ & $12.8 \%$ & $25.6 \%$ \\
\hline $\begin{array}{l}\text { Computadoras con conexión comple- } \\
\text { ta a Internet }\end{array}$ & $59.0 \%$ & $15.4 \%$ & $25.6 \%$ \\
\hline
\end{tabular}

Estas diferencias de acceso se reflejaron en el uso de los recursos electrónicos de información. El porcentaje de investigadores que leen listas de discusión es significativamente más alto entre los que trabajan en institutos (cerca del 30\% en los institutos, comparado con el $10 \%$ en los departamentos). La transferencia de archivos fue más frecuente entre investigadores de institutos ( $\mathrm{df}=88$, p01), y también ellos hicieron uso del correo electrónico más frecuentemente.

\section{CONCLUSIONES}

El acceso y uso de las computadoras entre los científicos mexicanos es generalmente bueno. Al parecer las universidades mexicanas hacen grandes esfuerzos por proveer a sus investigadores con tecnología de la información, y esta tecnología está siendo utilizada. La gran mayo ría de los científicos que contestaron esta encuesta tiene acceso a computadoras conectadas a Internet en sus lugares de trabajo, y la mayoría las 
usan para comunicarse por correo electrónico con sus colegas. Las conexiones fueron también menos usadas para acceder y distribuir información científica y publicaciones. Sin embargo, hay evidencias de que los problemas propios de la periferia, tales como preocupaciones con los costos y con la calidad de la conexión, persisten.

Se encontraron algunas diferencias individuales e institucionales en cuanto al acceso y uso de la tecnología de la información. Los investigadores con grado de doctor tienen mejor acceso a computadoras conectadas a las redes que quienes cuentan con grado de maestría; y también hacen más uso de las redes para establecer una comunicación formal e informal con sus colegas. Como grupo, los físicos tienen mejor acceso quelos biólogos a computadoras conectadas alas redes, y las usan más parala distribución de pre-prints y la transferencia de archivos. Finalmente, aquellos afiliados a institutos de investigación tienen mejor acceso a las computadoras, al correo electrónico y en general a Internet, y hacen más uso de sus aplicaciones que quienes trabajan en departamentos de universidades.

En cada caso, quienes tienen mejor acceso a las computadoras y/ o redes y hacen mayor uso de ellas (Ph.D .s, físicos, y aquellos que trabajan en institutos de investigación) también publican más en revistas extranjeras, lo cual sugiere que la actividad en la red puede, de alguna manera, estar relacionada con su participación en la ciencia internacional. Es razonable suponer que poder comunicarse fácilmente con colegas de otros países, tener un acceso fácil al trabajo de éstos, y hacer disponible su propio trabajo en forma electrónica, puede facilitar la participación en la ciencia internacional. ¿Q ué implicaciones tiene esto para quienes no están bien conectados, 0 están interesados en desarrollar áreas de investigación científica nacional (en contraste con áreas internacionales)?. Claramente este asunto amérita un estudio más detenido.

A unque los resultados aquí presentados pueden ser cuestionados debido al sesgo relacionado con el porcentaje de encuestados que trabajan en la UNAM, es importante mencionar que ARIE S registra el mayor número de proyectos e investigadores de esta universidad. Por otro lado existe también el riesgo de que los investigadores que contestaron la encuesta (tan sólo 26\% de la muestra de estudio) pueden ser quienes hacen un mayor uso de la tecnología de la información, pues serían ellos los más inclinados a interesarse por un cuestionario como el presente, así como a poseer un mejor conocimiento del tema. Aunque no se puede suponer que quienes contestaron esta encuesta son una muestra representativa de los investigadores mexicanos en los campos de la biología y la física, este estudio muestra algunos patrones interesantes sobre el uso dela tecnología de lainformación entre un grupo de científicos, y sugiere que el uso de la tecnología de la información puede estar relacionado con la internacionalización de la ciencia en los países en vías de desarrollo. 


\section{O BRAS CITA DAS}

Abels, E.G., Liebscher, P. y D enman, D.W. (1996). Factors that influence the use of electronic networks by science and engineering faculty at small institutions. Part I: Q ueries. Joumal of theAmerican Sociey for Information Saience, 47, 146-158.

Ainsworth, S. (1994). Mexican information resources in electronic format. En BonkerAnnual LibraryandBodk TradeAlmanac(pp.75-90). New Providence (N.J.): R.R. Bowker.

Al-Shanbari, H. y Meadows, A.J. (1995). Problems of communication and information-handling among scientists and engineers in Saudi universities. Jaumal of Information Sience 21, 473-478.

Almada deAscencio, M, y Pérez de Almada, S. Scholarly information and serials in Latin A merica: shifting political sands. TheSeialsLibranian, 21, 6985.

ARIES: proyetosypersonal deinvestigaaón(1998). Mexico City: Universidad Nacional Autónoma de México, D irección General de Intercambio Académico.

Asociación Nacional de Universidades e Instituciones de Educación Superior (ANUIES). (1998). Diretaria URL: http:/ / www.anuies.mx/ directorio/ Odirectorios.html [1998, diciembre 11].

Bishop, A.P. (1994). The role of computer networks in aerospace engineering. Library Trends 42, 694-729.

Borman, S. (1996). Is the web really worldwide? Cherical and Enginering Nens 74 (44), 35.

Cabezas, A. (1995). Internet: potential for services in Latin America. IFLA Jaumal, 21, 11-14.

Cabrajec, L., y D ukic, Z . (1991). Communication practices of Croatian scientists. Intemational Library Review, 23, 237-253.

Campos, E. M. (1997). Alternative media for getting information. Resaurce Sharingand Information Neworks 13, 57-76.

Cole, N.J. y Bawden, D . (1996). Bioinformatics in the pharmaceutical industry. Jaumal of Doumetation, 52, 51-68.

Cronin, B. y McKim, G . (1996). Science and scholarship on the World Wide Web: a North A merican perspective. Jaumal ofDoumentation, 52, 163-171.

De Ibarrola, M. (1992). Mexico. En B. Clark y G . Neave(Eds.),TheEngdope dia of Higher Education(pp.462-473). Oxford: Pergamon Press.

D elgado, H., y Russell, J.M. (1992). Impact of studies published in the international literature by scientists at the National University of Mexico. Scientomtrics, 23, 75-90. 
Ehikhamenor, F.A. (1990a). Informal scientific communication in Nigerian universities. Jaumal of theAmerianSodityforInfometionsaience, 41, 419-426.

Ehikhamenor, F.A. (1990b). The use of formal information sources in physical science research in Nigerian universities. Intemational Library Reiew 22, 149-161.

Elliot, R. (1997). The impact of electronic publishing on the scientific information chain. IFLA Jamal, 23, 351-355.

Fernández de Zamora, R.M. (1990). The economic crisis and the scientific, technical and cultural information services in Mexico. Intemational Library Review 22, 263-271.

- - -. (1991). Library resources in Latin America: ageneral panorama. IFLA Joumal, 17, 45-54.

Fortes, J. y Lomnitz, L.A. (1994). Becominga sientistin Mexica the dallenge of creatinga saientific community in an underdedeped country. University Park, PA: The Pennsylvania State University Press.

Gamboa, J.O A. (1998). Access to Latinamerican journals through the Internet: the option of Clase and Periodica databases. Ciencia da Infomacaa 27, 90-95.

Ginsparg, P. (1997). Winners and losers in the global research village. Seials Libraian, 30, 83-95.

Hurd, J.M. y Weller, A.C. (1997). From print to electronic: the adoption of information technology by academic chemists. SaieneandTehndogy Libraries 16, 147-70.

Internet Society. (1996). International connectivity. URL: http:/ / info.isoc.org/ images/ mapv15.gif. [1998, diciembre 11].

Krauskopf, M., Vera, M.I., Krauskopf, V., y Welljams-D orof, A. (1995). A citationist perspective on science in Latin America and the Caribbean, 1981-1993. Saientomerics 34, 3-25.

Lau, J. (1993). Research in the outskirts of science: the case of Mexico. Jaumal of Infomation and Library Reserench, 5, 39-46.

Lau, J. (1994). Information networking in Latin America: promises and challenges. In LatinA merica: theenerginginformationpower (Special Libraries Association State-of-the-Art Institute, Nov. 8-9, 1993) (pp.7-20). Washington, D.C.: Special Libraries Association.

Lau, J. y Castro, M. (1994). Computer and software for information services: an overview of Mexican progress. ResarceSharing and Infomation Netwakss 9, 91-100. 
Lazinger, S.S., Bar-Ilan, J. y Peritz, B.C. (1997). Internet use by faculty members in various disciplines: a comparative case study. Joumal of theAmrican Socidy for Information Saience, 48, 508-518.

Levison, A., eIglesias, H.R. (1993). The online industry in Mexico. Online, 17 (3), 116-119.

Licea de Arenas, J. (1990). Partial assessment of Mexican health sciences research 1982-1986. Saientantrics 23, 47-55.

Liebscher, P., Abels, E.G. y D enman, D.W. (1997). Factors that influence the use of electronic networks by science and engineering faculty at small institutions. Part II: Preliminary use indicators. Joumal of theAmericanSo didy for Information Saience 48, 496-507.

Magaña, P., Almeida, R.S., Carrollo T., C., Torres A., S. (1995). Divulgación de la ciencia en los medios escritos en México. En Cetto, A.M. y Hillerud, K. (E ditores). PublicadionescientíficasenAméicaLatima, pp.116-130. Mexico: International Council of Scientific Unions, UNESCO, UNAM, A cademia de la Investigación Científica, y Fondo de Cultura Económica.

Morton, H.C. y Price, A.J. (1988). TheACLSsurveyofschdars final reportofviens onpublications, computers, andlibraries Washington, D .C.: A merican Council of Learned Societies.

Network Wizards Internet D omain Survey (1998).

URL: http:/ / www.nw.com/ zone/ WWW/ top.html. [1998, diciembre $11]$.

Nkewe, K.M.C. (1995). Information methods of human and veterinary medical scientists (HVMS) in Borno State, Nigeria. Library and Information ScienceRerearch, 17, 41-48.

Nua Internet Surveys (1998). Business Week: web activity explodes in Latin America. URL: http:/ / www.nua.ie/ surveys/ index.cgi

Ornelas, C., y Levy, D.C. (1991). Mexico. En P. Altbach (Ed.), Intemational Higher Eduration: an Engdopedia (pp. 943-955). New York: G arland.

O sareh, F. y Wilson, C.S. (1997). Third world countries (TWC) research publications by disciplines: a country-by-country analysis. Scientomerics 39, 253-266.

Philip, G. (1995). Use of leading-edge' information systems by academic chemists in the U.K. Part I:T he results of a preliminary investigation. Jarrnal of Information Saience, 21, 187-199.

Rodríguez, K. (1993). The information search in Latin America: an analysis of Latin American databases. Libri, 43, 245-262.

Rodríguez, K. (1994). Barriers to information technology in Latin America and the Caribbean: some options. TheEletranicLibrary, 12, 29-35.

Rolinson, J., Al-Shanbari, H., y Meadows, A.J. (1996). Information usage by biological researchers. Jaumal of Infomation Science, 22, 47-53. 
Royal Society (1993). TheScientific, technical and meeical infomation systemintheU.K [London]: Royal Society, The British Library, and the Association of Learned and Professional Society Publishers.

Russell,J.M. (1995). The increasing role of international cooperation in scientific and technological research in Mexico. Sciento menics 34, 73-86.

- - -. (1998). Publishing patterns of Mexican scientists: differences between national and international papers. Säentonetrics, 41, 113-124.

Schoijet, M. y Worthington, R. (1993). Globalization of science and repression of scientists in Mexico. Scienee, Tehndogy and Human V alues 18, 209-230.

Sistema Nacional de Investigadores. (1998). Criteriosdeevaluacón URL: http:/ / www.conacyt.mx/ sni/ criterio.html. [1998, diciembre 11].

Sistema Nacional de Investigadores. (1998). Estadísticas básicas 1997-1998.

URL: http:// www.main.conacyt.mx/ sni/ estadisticas_1.html. [1998, diciembre 11].

Statistical Yearbook for LatinAmeicaand theCanibbean 1997. Santiago, Chile: Economic Commission on Latin America, 1998.

Tangeman, M. (1996). Competition calls. Infrastructurefinance 5 (7), 21-25.

UNESCO Statistical Yearbook 1997. Paris: UNESCO, 1998.

Voutssas, J. y Cetto, A.M. (1996). Electronic publishing: will it reach the whole world? In Electronic Publishing in Science (a joint ICSU Press/ UNESCO Conference). Paris, 19-23 February 1996. URL: http:/ / www.lmcp.jussieu.fr/ icsu/ Information/ Proc_0296/ voutssas.html.

World Bank (1997). WorddDedqumettRepat, 1997. Washington, D.C.: World Bank.
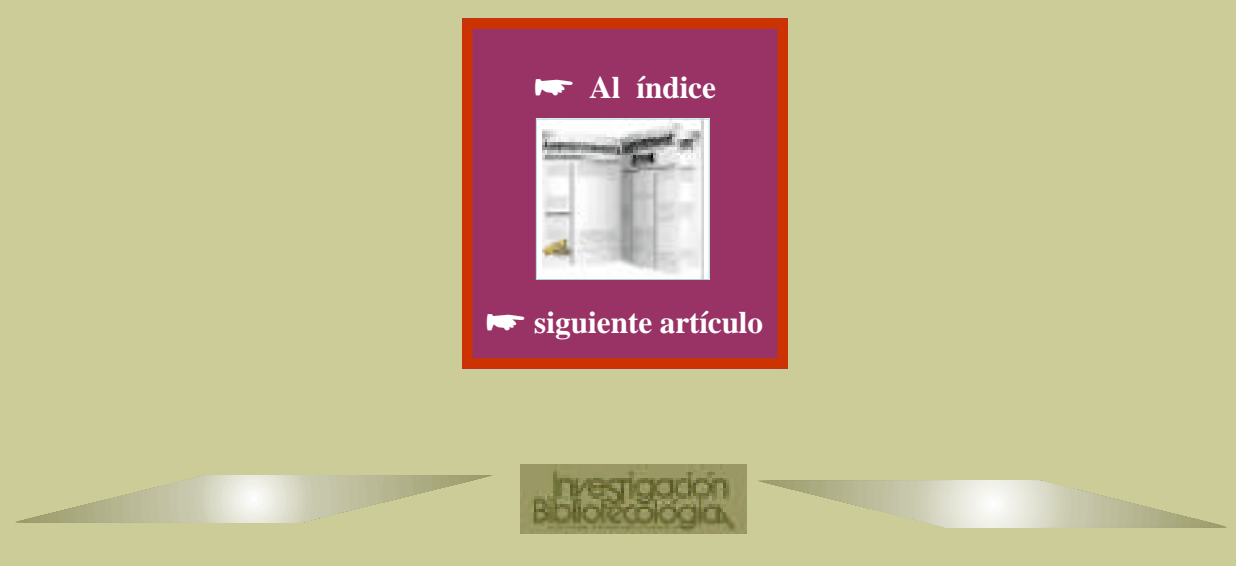